\title{
A phase I study of single-agent BEZ235 special delivery system sachet in Japanese patients with advanced solid tumors
}

\author{
Masanori Toyoda ${ }^{1} \cdot$ Koichiro Watanabe $^{2,5} \cdot$ Taro Amagasaki $^{3} \cdot$ Kazuto Natsume $^{3} \cdot$ Hiromi Takeuchi $^{3} \cdot$ Cornelia Quadt $^{4}$. \\ Kuniaki Shirao ${ }^{2} \cdot$ Hironobu Minami ${ }^{1}$ (1)
}

Received: 13 June 2018 / Accepted: 2 November 2018 / Published online: 16 November 2018

(c) The Author(s) 2018

\begin{abstract}
Purpose BEZ235 is a dual kinase inhibitor of phosphatidylinositol 3-kinase (PI3K) and mammalian target of rapamycin, which are key components of the PI3K pathway. This was an open-label, multicenter, dose-escalation, phase I study of single-agent BEZ235 in Japanese oncology patients to determine the maximum tolerated dose (MTD) of BEZ235 based on dose-limiting toxicities (DLTs).

Methods Dose escalation was guided by a standard 3+3 method and was based on DLTs observed in Cycle 1 and other safety, pharmacokinetic, and pharmacodynamic information. A total of 35 adult Japanese patients with advanced solid tumors received BEZ235 according to once daily (qd; $n=27)$ or twice daily (bid; $n=8)$ dosing schedules.

Results Two DLTs, namely, allergic reaction and thrombocytopenia, were observed at 1200 and $1400 \mathrm{mg}$ qd, respectively, while liver dysfunction was reported as a DLT at $400 \mathrm{mg}$ bid. The most common adverse events suspected to be related to BEZ235 in both dosing schedules were diarrhea, nausea, decreased appetite, stomatitis, and thrombocytopenia.

Conclusions Although the MTD was not established, the maximum clinically tolerable dose was determined to be $1200 \mathrm{mg}$ because two out of six patients required dose reduction in Cycle 2. The recommended dose was determined to be $1000 \mathrm{mg}$ qd, which was comparable with the results of the first-in-human BEZ235 study in Western patients with advanced solid tumors (NCT00620594). Additionally, the tolerability of BEZ235 $400 \mathrm{mg}$ bid in Japanese oncology patients was confirmed in this study.
\end{abstract}

ClinicalTrials.gov identifier NCT01195376.

Keywords PI3K $\cdot$ mTOR $\cdot$ Phase I $\cdot \mathrm{BEZ} 235 \cdot$ Inhibitor

\section{Introduction}

The phosphatidylinositol 3-kinase (PI3K) /mammalian target of rapamycin (mTOR) pathway is a key signal transduction systems linking multiple receptor tyrosine kinases and oncogenic proteins to essential cellular functions such as cell growth, survival, motility, and metabolism [1]. Important components of the signaling cascade include AKT, mTOR article (https://doi.org/10.1007/s00280-018-3725-2) contains supplementary material, which is available to authorized users.

Hironobu Minami

hminami@med.kobe-u.ac.jp

1 Division of Medical Oncology/Hematology, Department of Medicine, Kobe University Hospital, Kobe University Graduate School of Medicine, 7-5-1 Kusunoki-cho, Chuo-ku, Kobe 650-0017, Japan

2 Department of Medical Oncology and Hematology, Oita University Faculty of Medicine, Oita, Japan
Novartis Pharma K.K, Tokyo, Japan

4 Novartis Pharma AG, Basel, Switzerland

5 Present Address: Department of Medical Oncology, Kouseiren Tsurumi Hospital, Oita, Japan 
[2], and phosphatase and tensin homolog (PTEN), a negative regulator of PI3K [3]. Several lines of evidence suggest that constitutive PI3K activation is a critical step in mediating the transforming potential and growth stimulating activity of various oncogenes and tumor suppressors, thereby contributing to the onset and growth of many solid tumors as well as tumors of the hematopoietic system [4, 5].

At low exposures, BEZ235 is a catalytic inhibitor of mTOR, inhibiting both mTORC1 and mTORC2 kinases, whereas at high exposures, which were expected at the doses tested in this study, BEZ235 inhibits mTOR as well as all class I PI3Ks. The antitumor activity of BEZ235 was demonstrated in preclinical studies [6].

Several formulations of BEZ235, such as a hard gelatin capsule formulation and a special delivery system (SDS) formulation provided as capsules or granules in a sachet, were investigated in the first-in-human (FIH) study of BEZ235 [7]. The SDS formulations were developed to decrease pharmacokinetic (PK) variability and improve bioavailability. However, at the doses of $400 \mathrm{mg}$ and higher tested in this study, the SDS capsule still showed high inter-patient variability, but the PK profile of the SDS sachet formulation appeared more consistent. Here, we report the results of a phase I study to investigate the safety, tolerability, preliminary efficacy, PK, and maximum tolerated dose (MTD) of BEZ235 SDS granules in a sachet formulation in Japanese patients. To reduce the risk of gastrointestinal toxicities observed with the once daily (qd) dosing regimen, twice daily (bid) dosing of BEZ235 was also investigated.

\section{Materials and methods}

\section{Study oversight}

This study was designed as a phase I, multicenter, open-label trial in which BEZ235 SDS sachet was administered orally as a single agent on a continuous qd or bid dosing schedule in Japanese patients with advanced solid malignancies (ClinicalTrials.gov Identifier: NCT01195376). The study was designed by the sponsor (Novartis Pharmaceuticals Corporation) and performed in accordance with the principles of Good Clinical Practice. The protocol was approved by an Institutional Review Board at each institution and the study was conducted according to the ethical principles of the Declaration of Helsinki. Informed consent was obtained from all individual participants included in the study.

\section{Patient selection}

Key inclusion criteria were as follows: patients with histologically confirmed, advanced, unresectable solid tumors who have progressed on (or were not able to tolerate) standard therapy or for whom no standard anticancer therapy existed; at least one measurable lesion as defined by the Response Evaluation Criteria in Solid Tumors (RECIST) v1.0; age $\geq 20$ years; Eastern Cooperative Oncology Group performance status (ECOG PS) 0-2; and life expectancy of at least 12 weeks.

Key exclusion criteria were as follows: patients with brain tumor and/or brain metastases, with signs/symptoms potentially attributable to brain metastases or if brain lesions could not be ruled out by radiologic imaging, fulminant disease including fulminant hepatitis, prior treatment with a PI3K inhibitor, acute or chronic liver disease, clinically relevant renal disease, acute or chronic pancreatitis, history or presence of interstitial lung disease (ILD), peripheral neuropathy $\geq$ Common Terminology Criteria for Adverse Events (CTCAE) grade 2, and unresolved diarrhea $\geq$ CTCAE grade 2 .

\section{Study objectives}

The primary objective was to determine the MTD of BEZ235 as a single agent based on the incidence rate of dose-limiting toxicities (DLTs) when administered orally. The secondary objectives were to assess the safety and tolerability of BEZ235, characterize its PK profiles, evaluate preliminary efficacy, and assess potential treatment effects on the pharmacodynamic (PD) biomarkers of the PI3K/ mTOR pathway.

\section{Study design and treatment plan}

BEZ235 was administered orally as a single agent on a continuous qd or bid dosing schedule. The starting dose level for the first cycle (28 days) was set as $400 \mathrm{mg} /$ day qd, based on the FIH BEZ235 study in Western patients (NCT00620594). In this study, no study drug-related $\geq$ grade 2 adverse events (AEs) were observed during Cycle 1 with the SDS capsule at $400 \mathrm{mg}$ qd. At the $800 \mathrm{mg}$ qd dose level, one DLT (grade 3 fatigue) was observed and at the $1000 \mathrm{mg}$ qd dose level, two DLTs (grade 3 fatigue and skin rash, which required more than 7 days treatment interruption) were observed. The MTD for the BEZ235 SDS capsule was established at $1000 \mathrm{mg}$ qd [8]. Furthermore, in the previous study, a granule formulation (BEZ235 SDS sachet) was developed because of difficulties in swallowing the large size capsules of the investigational drug. The ingredients of this new formulation, which are dispensed as granules contained within a sachet, are essentially the same as the capsule. Therefore, it was anticipated that the PK profiles would not differ to an extent that would significantly affect the dosing of the sachet formulation. The starting dose of $400 \mathrm{mg}$ qd was half of the dose level of the BEZ235 SDS capsule with the first DLT and also less than half of the MTD of the BEZ235 
SDS capsule. The standard $3+3$ method for dose escalation was used to establish the MTD of BEZ235. Decisions regarding dose escalation were based on the safety profile, particularly DLTs, observed in the first cycle of treatment for each patient.

\section{Safety assessments}

Safety assessments consisted of monitoring and recording of all AEs, including laboratory abnormalities and vital signs. DLTs were defined as an AE or a laboratory abnormality assessed as related to the study drug and occurring within the first 28 days of treatment (Cycle 1), as defined in Supplemental Table S1.

\section{Response assessments}

Tumor lesions were assessed by the investigators according to the RECIST guidelines, v1.0. Patients underwent screening using computed tomography (CT) or magnetic resonance imaging (MRI) scans. Screening was performed within 28 days of the first dose. The post-baseline RECIST assessments were performed every two cycles and then at the end of treatment.

\section{Pharmacokinetics}

Blood samples for plasma concentration-time profiles of BEZ235 and its relevant metabolites were collected from all patients. The plasma samples were assayed for BEZ235 concentrations using a validated liquid chromatography-tandem mass spectrometry assay (LC-MS/MS). The PK parameters were determined by non-compartmental analysis using Phoenix WinNonlin 6.2 ${ }^{\circledR}$ (Pharsight, Mountain View, CA). The following PK parameters were summarized as the primary PK parameters for BEZ235 and its metabolite(s): $\mathrm{AUC}_{\text {last }}$, $\mathrm{AUC}_{0-24}, \mathrm{AUC}_{0-12}, \mathrm{AUC}_{\text {inf }}, C_{\max }$, and $T_{\max }$.

\section{Biomarkers}

Normal skin samples were collected at baseline and the end of Cycle 1 to assess the effect of BEZ235 on the PI3K/ mTOR pathway based on the level of S6 phosphorylation (pS6). Tumor samples were analyzed at a qualified laboratory for confirming the gene status of PIK3CA and PTEN.

\section{Results}

\section{Patient characteristics}

Between 06-Oct-2010 and 03-Jul-2013, 35 patients were enrolled and received at least one dose of BEZ235 with either the qd $(n=27)$ or bid $(n=8)$ dosing schedule. The baseline demographic and patient characteristics data are presented in Table 1. Patients with various types of cancers were enrolled, and all patients except one patient with carcinoid of the rectum were heavily pretreated before enrollment.

\section{Safety}

Among the 27 patients enrolled in the qd group, 24 patients were included in the dose-determining analysis set $(400 \mathrm{mg}$, $n=3 ; 800 \mathrm{mg}, n=3 ; 1000 \mathrm{mg}, n=6 ; 1200 \mathrm{mg}, n=6$; and $1400 \mathrm{mg}, n=6$ ). Three patients were excluded because of the following reasons: did not receive the required minimum number of BEZ235 doses in Cycle 1 due to disease progression (1000 mg group), AE that did not meet the DLT criteria (1000 mg group), and withdrawal of consent $(1400 \mathrm{mg}$ group). One out of the six evaluable patients in the 1200 and $1400 \mathrm{mg}$ qd groups each experienced DLTs of grade 2 allergic reaction and grade 4 thrombocytopenia, respectively (Table 2). In one patient who experienced allergic reaction in the $1200 \mathrm{mg}$ qd group, the reaction was accompanied with grade 1 fever and grade 3 rash that spread suddenly when the patient restarted the study treatment after interruption. The study treatment had to be discontinued in this patient due to this event. Therefore, this allergic reaction was considered as a DLT, although it was not prespecified in the protocol definitions of DLT (Online supplement Table S1).

Even though a DLT occurred in only one out of the six patients at $1400 \mathrm{mg} \mathrm{qd}$, the dose was not escalated to more than $1400 \mathrm{mg}$, considering that grade $3 / 4$ thrombocytopenia was observed in one patient at $1400 \mathrm{mg}$. Furthermore, dose interruption due to AEs was required in six patients, and three patients required dose reduction in early cycles. Therefore, the MTD, which is the highest drug dose that causes DLT in no more than one out of six patients in Cycle 1, was not reached. While the rates of DLTs were similar at both $1200 \mathrm{mg}$ qd and $1400 \mathrm{mg} \mathrm{qd}$, the safety of $1400 \mathrm{mg}$ qd was considered less favorable. As described above, severe (grade 3/4) thrombocytopenia, dose interruptions in Cycle 1 and dose reductions after Cycle 1 were more frequently observed in patients treated with $1400 \mathrm{mg}$ than $1200 \mathrm{mg}$. Therefore, $1200 \mathrm{mg}$ was determined as the maximum clinically tolerable dose of BEZ235 in a qd dosing schedule, while $1000 \mathrm{mg}$ was determined as the recommended dose (RD) because it was the highest dose that did not cause any DLT.

Eight patients were treated with BEZ235 at a dose of $400 \mathrm{mg}$ bid (Table 2). Of these, three patients were excluded from the dose-determining analysis set because they had not received the required minimum number (i.e., bid for 21 days) of BEZ235 doses in Cycle 1 for DLT evaluation due to an AE that did not meet the DLT criteria. However, one out of the three excluded patients was considered as clinically 
Table 1 Patient demographics and disease characteristics based on treatment group

\begin{tabular}{|c|c|c|c|c|c|c|c|}
\hline \multirow[t]{2}{*}{ Characteristics } & \multicolumn{6}{|c|}{ BEZ235 qd $(N=27)$} & \multirow{2}{*}{$\begin{array}{l}\text { BEZ235 bid } \\
400 \mathrm{mg}(N=8)\end{array}$} \\
\hline & $400 \mathrm{mg}(n=3)$ & $800 \mathrm{mg}(n=3)$ & $1000 \mathrm{mg}(n=8)$ & $1200 \mathrm{mg}(n=6)$ & $1400 \mathrm{mg}(n=7)$ & $\begin{array}{l}\text { All patients } \\
(N=27)\end{array}$ & \\
\hline $\begin{array}{l}\text { Age, years, } \\
\text { median (range) }\end{array}$ & $65(52-69)$ & $62(61-74)$ & $59(31-70)$ & $65(37-67)$ & $55(38-74)$ & $62(31-74)$ & $50(36-74)$ \\
\hline \multicolumn{8}{|l|}{$\mathrm{Sex}, N(\%)$} \\
\hline Male & 0 & $2(66.7)$ & $8(100)$ & $4(66.7)$ & $2(28.6)$ & $16(59.3)$ & $4(50.0)$ \\
\hline Female & $3(100)$ & $1(33.3)$ & 0 & $2(33.3)$ & $5(71.4)$ & $11(40.7)$ & $4(50.0)$ \\
\hline \multicolumn{8}{|l|}{ ECOG PS, $N(\%)$} \\
\hline 0 & $2(66.7)$ & 0 & $4(50.0)$ & $3(50.0)$ & $2(28.6)$ & $11(40.7)$ & $3(37.5)$ \\
\hline 1 & $1(33.3)$ & $3(100)$ & $4(50.0)$ & $3(50.0)$ & $5(71.4)$ & $16(59.3)$ & $5(62.5)$ \\
\hline \multicolumn{8}{|c|}{ Primary site of cancer, $N(\%)$} \\
\hline Rectum & 0 & 0 & $2(25.0)$ & $1(16.7)$ & $2(28.6)$ & $5(18.5)$ & $1(12.5)$ \\
\hline Lung & 0 & 0 & $1(12.5)$ & 0 & $2(28.6)$ & $3(11.1)$ & 0 \\
\hline Pancreas & 0 & $1(33.3)$ & 0 & 0 & $1(14.3)$ & $2(7.4)$ & $2(25.0)$ \\
\hline Colon & 0 & 0 & 0 & $2(33.3)$ & 0 & $2(7.4)$ & $1(12.5)$ \\
\hline Bone & $1(33.3)$ & 0 & 0 & 0 & $1(14.3)$ & $2(7.4)$ & $1(12.5)$ \\
\hline Soft tissue & 0 & 0 & $2(25.0)$ & 0 & 0 & $2(7.4)$ & 0 \\
\hline Stomach & 0 & 0 & $1(12.5)$ & 0 & 0 & $1(3.7)$ & $2(25.0)$ \\
\hline Other & $1(33.3)$ & $2(66.7)$ & $1(12.5)$ & $1(16.7)$ & $1(14.3)$ & $6(22.2)$ & $1(12.5)$ \\
\hline Unknown & $1(33.3)$ & 0 & $1(12.5)$ & $2(33.3)$ & 0 & $4(14.8)$ & 0 \\
\hline \multicolumn{8}{|c|}{ Number of prior regimens, $N(\%)$} \\
\hline 0 & 0 & 0 & 0 & 0 & $1(14.3)$ & $1(3.7)$ & 0 \\
\hline $1-2$ & $3(100)$ & $2(66.7)$ & $2(25.0)$ & $4(66.7)$ & $3(42.9)$ & $14(51.9)$ & $3(37.5)$ \\
\hline $3-4$ & 0 & 0 & $6(75.0)$ & $1(16.7)$ & $1(14.3)$ & $8(29.6)$ & $3(37.5)$ \\
\hline$\geq 5$ & 0 & $1(33.3)$ & 0 & $1(16.7)$ & $2(28.6)$ & $4(14.8)$ & $2(25.0)$ \\
\hline
\end{tabular}

bid twice daily, ECOG PS Eastern Cooperative Oncology Group Performance Status, $q d$ once daily

Table 2 Summary of patient treatment and DLTs

\begin{tabular}{llllll}
\hline Regimen & Dose $(\mathrm{mg})$ & Full analysis set & $\begin{array}{l}\text { Dose-determining } \\
\text { analysis set }\end{array}$ & $\begin{array}{l}\text { Number of patients } \\
\text { who experienced a } \\
\text { DLT }\end{array}$ & $\begin{array}{l}\text { DLT } \\
\text { BEZ235 qd }(N=27)\end{array}$ \\
& 400 & 3 & 3 & 0 & - \\
& 100 & 3 & 3 & 0 & - \\
& 1200 & 6 & 6 & 1 & - \\
BEZ235 bid $(N=8)$ & 1400 & 7 & 6 & 1 & Grade 2 allergic reaction \\
& 400 & 8 & 6 & 1 & Grade 4 thrombocytopenia \\
\hline
\end{tabular}

bid twice daily, DLT dose-limiting toxicity, $q d$ once daily

relevant for the evaluation of DLT. This patient suspended drug administration from the second dosing of Cycle 1 Day 14 to the first dosing of Day 21, resulting in a total of 14 skipped doses (corresponding to 7 days). Therefore, this patient was considered as evaluable for the assessment of safety and tolerability based on the discussion between the investigators and the sponsor. A total of six patients, including this patient, were evaluated for the determination of
DLTs. Only one out of six patients in the $400 \mathrm{mg}$ bid cohort experienced a DLT of grade 2 liver dysfunction (Table 2). This patient had adenocarcinoma of the rectum with liver metastases and elevated levels of total bilirubin, alkaline phosphatase, and gamma-GTP documented during screening. These values continued to increase when the patient restarted treatment after dose interruption, regardless of dose reduction. Therefore, these abnormal values were considered 
to be liver dysfunction related to the study drug and were evaluated as a DLT, although each abnormal value did not meet the DLT criteria for hepatic toxicities. In conclusion, $400 \mathrm{mg}$ bid was considered as a tolerable dose.

The median duration of exposure was 56 (2-280) days for the qd and 43.5 (21-115) days for the bid dosing schedules (Table 3). All patients in both dosing groups experienced at least one AE. In the qd group, the most frequently occurring AEs were diarrhea, decreased appetite, nausea, stomatitis, fatigue, and vomiting. In the bid group, the most frequently occurring AEs were decreased appetite, diarrhea, nausea, stomatitis, fatigue, and thrombocytopenia. Most of the patients in both groups experienced diarrhea, decreased appetite, and nausea (Tables 4, 5).

Grade 3 or 4 AEs were reported in 18 patients (66.7\%) in the qd dosing and seven patients $(87.5 \%)$ in the bid dosing groups. In the qd group, grade 3 or 4 AEs suspected to be study drug-related reported in more than one patient were lymphopenia [four patients (14.8\%)], neutropenia and fatigue [three patients $(11.1 \%)$ each], and thrombocytopenia and rash [two patients (7.4\%) each]. In the bid group, grade 3 or 4 AEs suspected to be study drug-related included thrombocytopenia and lymphopenia, which were reported in two patients (25.0\%) each and all other grade 3 or 4 AEs occurred in a single patient.

No patient died during the study period, including a 30-day follow-up. Study drug-related serious adverse events (SAEs) were reported for only two patients in the qd dosing group, namely, grade 2 colitis (1000 mg) and grade 2 Pneumocystis jiroveci pneumonia $(1400 \mathrm{mg})$. In the bid dosing group, one patient experienced a study drug-related SAE of grade 4 diarrhea.

The most frequent reason for treatment discontinuation was disease progression [16 patients (59.3\%) with qd dosing and seven patients (87.5\%) with bid dosing]. In the qd group, seven patients (25.9\%) discontinued the study drug due to AEs. In the bid group, an AE leading to study drug discontinuation was reported in one patient $(12.5 \%)$. This rectal cancer patient who experienced a DLT of grade 2 liver dysfunction discontinued the study drug in consideration of the balance between benefit with the study medicine and the risk of abnormal hepatic function, as the DLT was suspected to be study drug-related.

\section{Efficacy}

All 35 patients were evaluable for response per RECIST v1.0 guidelines. No complete or partial responses (CR or PR) were reported across all dose levels in any of the dosing schedule groups. Stable disease (SD) was observed as the best response in 14 of 27 (51.9\%) evaluable patients with a mean duration of study treatment exposure of 75.3 days and 2 of $8(25.0 \%)$ evaluable patients with a mean duration of study treatment exposure of 55.9 days in the qd and bid dosing groups, respectively. One patient with carcinoma of unknown primary who was evaluated as having SD with continued BEZ235 qd treatment for 10 months.

\section{Pharmacokinetics}

In general, the $C_{\max }$ and area under the curve (AUC) increased with dose on Cycle 1 Day 1 and Day 8, though no clear trend was observed on Day 28. For both the qd and bid regimens, PK exposures on Day 8 were at least threefold higher than on Day 1, whereas exposures on Day 8 and Day 28 were comparable. Based on these findings, the steadystate was expected to be achieved at least on Day 8. At these oncology doses, the inter-individual variability was high,

Table 3 Overall exposure

\begin{tabular}{|c|c|c|c|c|c|c|c|}
\hline \multirow{2}{*}{ Overall exposure } & \multicolumn{6}{|l|}{ BEZ235 qd } & \multirow{2}{*}{$\begin{array}{l}\text { BEZ235 bid } \\
400 \mathrm{mg}(N=8)\end{array}$} \\
\hline & $400 \mathrm{mg}(n=3)$ & $800 \mathrm{mg}(n=3)$ & $1000 \mathrm{mg}(n=8)$ & $1200 \mathrm{mg}(n=6)$ & $1400 \mathrm{mg}(n=7)$ & All patients $(N=27)$ & \\
\hline \multicolumn{8}{|l|}{ Exposure (weeks) } \\
\hline$\leq 4$ & 0 & 0 & $3(37.5 \%)$ & $2(33.3 \%)$ & $1(14.3 \%)$ & $6(22.2 \%)$ & $3(37.5 \%)$ \\
\hline$>4$ to $\leq 8$ & $2(66.7 \%)$ & 0 & $2(25.0 \%)$ & $2(33.3 \%)$ & $2(28.6 \%)$ & $8(29.6 \%)$ & $2(25.0 \%)$ \\
\hline$>8$ to $\leq 12$ & 0 & 0 & $2(25.0 \%)$ & 0 & $1(14.3 \%)$ & $3(11.1 \%)$ & $1(12.5 \%)$ \\
\hline$>12$ & $1(33.3 \%)$ & $3(100.0 \%)$ & $1(12.5 \%)$ & $2(33.3 \%)$ & $3(42.9 \%)$ & $10(37.0 \%)$ & $2(25.0 \%)$ \\
\hline \multicolumn{8}{|c|}{ Duration of study treatment exposure (days) } \\
\hline$n$ & 3 & 3 & 8 & 6 & 7 & 27 & 8 \\
\hline Mean & 52.7 & 149.7 & 48.8 & 82.3 & 77.4 & 75.3 & 55.9 \\
\hline SD & 28.99 & 31.77 & 30.78 & 100.31 & 52.23 & 62.36 & 37.72 \\
\hline Median & 44.0 & 167.0 & 37.0 & 39.5 & 68.0 & 56.0 & 43.5 \\
\hline Minimum & 29.0 & 113.0 & 19.0 & 16.0 & 2.0 & 2.0 & 21.0 \\
\hline Maximum & 85.0 & 169.0 & 112.0 & 280.0 & 169.0 & 280.0 & 115.0 \\
\hline
\end{tabular}

bid twice daily, $q d$ once daily, $S D$ standard deviation 


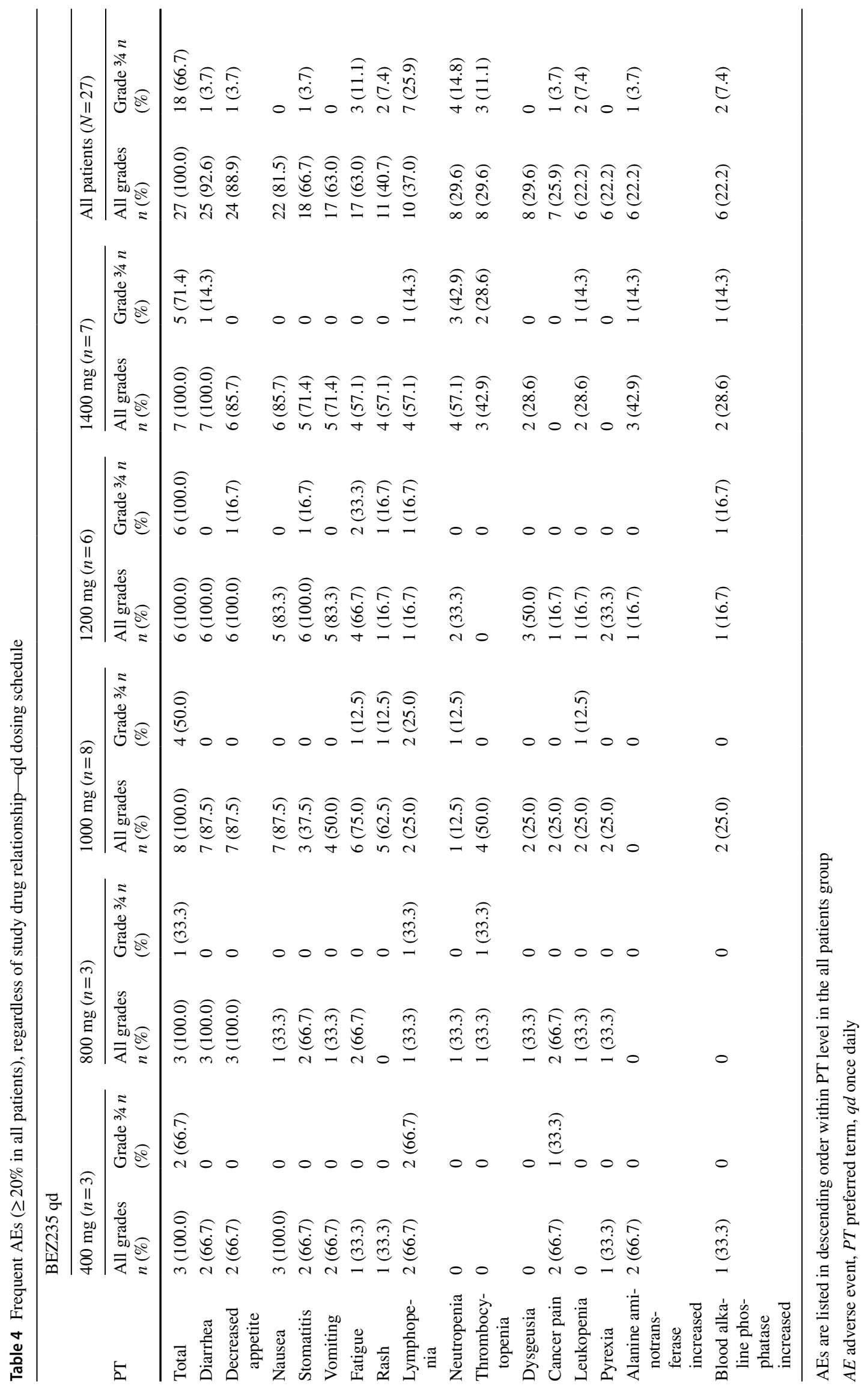


Table 5 Frequent AEs ( $\geq 20 \%$ in all patients), regardless of study drug relationship-bid dosing schedule

\begin{tabular}{|c|c|c|}
\hline \multirow[b]{3}{*}{ PT } & \multicolumn{2}{|l|}{ BEZ235 bid } \\
\hline & \multicolumn{2}{|l|}{$400 \mathrm{mg} N=8$} \\
\hline & All grades $n(\%)$ & Grade $3 / 4 n(\%)$ \\
\hline Total & $8(100.0)$ & $7(87.5)$ \\
\hline Diarrhea & $7(87.5)$ & $1(12.5)$ \\
\hline Nausea & $7(87.5)$ & 0 \\
\hline Decreased appetite & $7(87.5)$ & $1(12.5)$ \\
\hline Thrombocytopenia & $5(62.5)$ & $2(25.0)$ \\
\hline Stomatitis & $5(62.5)$ & 0 \\
\hline Fatigue & $5(62.5)$ & $1(12.5)$ \\
\hline Lymphopenia & $4(50.0)$ & $2(25.0)$ \\
\hline Vomiting & $4(50.0)$ & 0 \\
\hline $\begin{array}{l}\text { Alanine aminotransferase } \\
\text { increased }\end{array}$ & $4(50.0)$ & 0 \\
\hline $\begin{array}{l}\text { Aspartate aminotransferase } \\
\text { increased }\end{array}$ & $4(50.0)$ & $1(12.5)$ \\
\hline Haemoglobin decreased & $4(50.0)$ & $1(12.5)$ \\
\hline Insomnia & $4(50.0)$ & 0 \\
\hline Dry skin & $4(50.0)$ & 0 \\
\hline Rash & $4(50.0)$ & 0 \\
\hline Hypoalbuminaemia & $4(50.0)$ & $1(12.5)$ \\
\hline Cheilitis & $3(37.5)$ & 0 \\
\hline Dysphagia & $3(37.5)$ & 0 \\
\hline Cancer pain & $3(37.5)$ & $2(25.0)$ \\
\hline Blood creatinine increased & $3(37.5)$ & 0 \\
\hline Malaise & $2(25.0)$ & $1(12.5)$ \\
\hline Pyrexia & $2(25.0)$ & 0 \\
\hline Urinary tract infection & $2(25.0)$ & 0 \\
\hline $\begin{array}{l}\text { Blood alkaline phosphatase } \\
\text { increased }\end{array}$ & $2(25.0)$ & 0 \\
\hline $\begin{array}{l}\text { Gamma-glutamyltransferase } \\
\text { increased }\end{array}$ & $2(25.0)$ & 0 \\
\hline Hyperkalaemia & $2(25.0)$ & 0 \\
\hline Hyponatraemia & $2(25.0)$ & $1(12.5)$ \\
\hline Anxiety & $2(25.0)$ & 0 \\
\hline
\end{tabular}

AEs are listed in descending order within PT level

$A E$ adverse event, bid twice daily, $P T$ preferred term

with a coefficient of variation greater than $100 \%$ on the AUC and $C_{\max }$ at most of the visits and doses. Median $\mathrm{T}_{\max }$ was approximately $4 \mathrm{~h}$ across all dose levels and dosing schedules (individual $\mathrm{T}_{\max }$ range, 1.0-24.0 $\mathrm{h}$ ) and was similar on Day 1, Day 8, and Day 28 of Cycle 1 . The results are consistent with the result of SDS sachet evaluated in the FIH study of BEZ235 in Western Patients [9]. Due to the long $T_{\max }$, the elimination half-life $\left(T_{1 / 2}\right)$ was not well characterized because of the absence of samples collected between 8 and 24 h post-dose (Table 6). Overall, the $C_{\max }$ and AUC appeared to increase in a dose-dependent manner.

\section{Biomarkers}

Sequencing results were obtained for nine tumor samples; no mutations of PIK3CA or PTEN were detected. One tumor sample was declared PTEN-negative, and in another patient, $40 \%$ of the tumor cells were PTEN-negative. Because of administrative problems, limited analysis was done from the collected small number of samples; no correlation with the clinical efficacy was observed and no further conclusions could be drawn.

Analysis of pS6 in normal skin was performed in 6 patients treated with $400 \mathrm{mg}$ bid. Reduction was observed in $50 \%$ of the samples (3/6). Two of these three patients had SD until Cycle 4 or 5 with dose reductions up to $200 \mathrm{mg}$.

\section{Discussion}

This study evaluated the safety and tolerability of BEZ235 doses from $400 \mathrm{mg}$ to $1400 \mathrm{mg}$ in Japanese patients with solid tumors. Each one out of six evaluable patients experienced DLTs of grade 2 allergic reaction at the $1200 \mathrm{mg}$ dose level and grade 4 thrombocytopenia at the $1400 \mathrm{mg}$ dose level. Thrombocytopenia was also reported as a DLT but allergic reaction was not reported as DLT in the FIH study in Western oncology patients. The patient diagnosed with allergic reaction had developed grade 1 rash and stomatitis. The study treatment was resumed after AEs were resolved, and on the same day, the patient experienced grade 1 fever. Fever was resolved with medication; however, on the next day, grade 3 rash was developed. These events of fever and rash that spread suddenly when study treatment was resumed, were judged as allergic reaction. Fever and rash were frequently reported AEs in different clinical studies of BEZ235, but there is no information about worsening of these events after re-exposure to study treatment. The single occurrence of this allergic reaction as DLT in our study does not allow conclusions about ethnic differences in the safety profile of BEZ235.

While the rates of DLTs were similar at both $1200 \mathrm{mg}$ qd and $1400 \mathrm{mg}$ qd, the safety of $1400 \mathrm{mg}$ qd was considered less favorable (Table 2). Although the frequency of the DLT did not fulfil the criteria of the MTD prespecified in the protocol (the highest drug dose that causes DLT in no more than one out of six patients in Cycle 1), the maximum clinically tolerable dose of single-agent BEZ235 at a qd dosing schedule was determined to be $1200 \mathrm{mg}$ qd, considering the observed toxicities and dose reductions in Cycle 2. The dose of $1000 \mathrm{mg}$ qd was considered as the RD because it was the highest dose that did not cause any DLT.

In the FIH study of BEZ235 SDS sachet in Western oncology patients, only one DLT (grade 3 thrombocytopenia) was observed at $1600 \mathrm{mg}$; however, $50 \%$ of the patients 
Table 6 Summary of the primary PK parameters of BEZ235

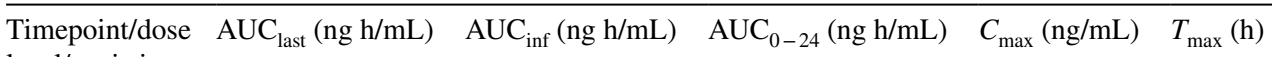
level/statistics

\begin{tabular}{|c|c|c|c|c|c|}
\hline \multicolumn{6}{|c|}{ Cycle 1 Day 1} \\
\hline \multicolumn{6}{|l|}{$400 \mathrm{mg}$ qd } \\
\hline$n$ & 3 & 2 & 3 & 3 & 3 \\
\hline Median & 1036 & 978 & 1038 & 135 & 4.0 \\
\hline Min/Max & $821 / 13,782$ & $895 / 1061$ & $821 / 13,869$ & $69.9 / 1000$ & $4.0 / 4.0$ \\
\hline \multicolumn{6}{|l|}{$800 \mathrm{mg} \mathrm{qd}$} \\
\hline$n$ & 3 & 1 & 3 & 3 & 3 \\
\hline Median & 2558 & 1488 & 2558 & 154 & 8.0 \\
\hline Min/Max & $1433 / 6096$ & $1488 / 1488$ & $1438 / 6096$ & $150 / 466$ & $4.0 / 8.0$ \\
\hline \multicolumn{6}{|l|}{$1000 \mathrm{mg} \mathrm{qd}$} \\
\hline$n$ & 6 & 3 & 4 & 6 & 6 \\
\hline Median & 4492 & 1407 & 4334 & 472 & 5.0 \\
\hline Min/Max & $869 / 19,292$ & $890 / 7301$ & $869 / 19,265$ & $106 / 970$ & $1.0 / 8.0$ \\
\hline \multicolumn{6}{|l|}{$1200 \mathrm{mg}$ qd } \\
\hline$n$ & 5 & 1 & 3 & 5 & 5 \\
\hline Median & 6420 & 2292 & 6417 & 403 & 6.0 \\
\hline Min/Max & $2261 / 25,472$ & $2292 / 2292$ & $2264 / 15,775$ & $203 / 1500$ & $4.0 / 23.4$ \\
\hline \multicolumn{6}{|l|}{$1400 \mathrm{mg} \mathrm{qd}$} \\
\hline$n$ & 5 & 1 & 2 & 5 & 5 \\
\hline Median & 10,224 & 1286 & 13,736 & 733 & 8.0 \\
\hline Min/Max & $1208 / 37,073$ & $1286 / 1286$ & $1215 / 26,258$ & $87.7 / 1940$ & $4.0 / 24.0$ \\
\hline \multicolumn{6}{|l|}{$400 \mathrm{mg}$ bid } \\
\hline$n$ & 7 & 2 & 4 & 7 & 7 \\
\hline Median & 2283 & 1995 & 1755 & 301 & 6.0 \\
\hline Min/Max & $606 / 7719$ & $1402 / 2587$ & $606 / 5450$ & $84.6 / 1150$ & $1.0 / 8.0$ \\
\hline \multicolumn{6}{|c|}{ Cycle 1 Day 8} \\
\hline \multicolumn{6}{|l|}{$400 \mathrm{mg} \mathrm{qd}$} \\
\hline$n$ & 3 & 2 & 3 & 3 & 3 \\
\hline Median & 7349 & 14,315 & 7351 & 754 & 4.0 \\
\hline Min/Max & $4311 / 17,295$ & $7657 / 20,974$ & $4310 / 17,465$ & $314 / 1470$ & $4.0 / 8.0$ \\
\hline \multicolumn{6}{|l|}{$800 \mathrm{mg} q d$} \\
\hline$n$ & 3 & 1 & 3 & 3 & 3 \\
\hline Median & 17,327 & 19,866 & 17,327 & 1170 & 6.0 \\
\hline Min/Max & $8497 / 19,617$ & $19,866 / 19,866$ & $8497 / 19,626$ & $609 / 1700$ & $4.0 / 8.0$ \\
\hline \multicolumn{6}{|l|}{$1000 \mathrm{mg}$ qd } \\
\hline$n$ & 7 & 3 & 3 & 7 & 7 \\
\hline Median & 9347 & 8301 & 8261 & 912 & 6.0 \\
\hline Min/Max & $482 / 46,393$ & $496 / 9469$ & $482 / 9347$ & $76.1 / 2850$ & $1.0 / 8.0$ \\
\hline \multicolumn{6}{|l|}{$1200 \mathrm{mg} \mathrm{qd}$} \\
\hline$n$ & 4 & 1 & 4 & 4 & 4 \\
\hline Median & 33,484 & 14,438 & 34,179 & 1810 & 4.0 \\
\hline Min/Max & $12,760 / 46,522$ & $14,438 / 14,438$ & $12,840 / 46,522$ & $1130 / 2410$ & $2.0 / 4.0$ \\
\hline \multicolumn{6}{|l|}{$1400 \mathrm{mg} \mathrm{qd}$} \\
\hline$n$ & 5 & 0 & 3 & 5 & 5 \\
\hline Median & 35,882 & - & 36,375 & 2480 & 4.0 \\
\hline Min/Max & $13,688 / 74,490$ & - & $26,544 / 40,807$ & $720 / 3910$ & $3.9 / 7.9$ \\
\hline \multicolumn{6}{|l|}{$400 \mathrm{mg}$ bid } \\
\hline$n$ & 6 & 1 & 5 & 6 & 6 \\
\hline Median & 9702 & 2786 & 10,677 & 1046 & 4.0 \\
\hline Min/Max & $2616 / 52,196$ & $2786 / 2786$ & $2616 / 53,881$ & $455 / 6070$ & $2.0 / 12.0$ \\
\hline
\end{tabular}


Table 6 (continued)

\begin{tabular}{|c|c|c|c|c|c|}
\hline $\begin{array}{l}\text { Timepoint/dose } \\
\text { level/statistics }\end{array}$ & $\mathrm{AUC}_{\text {last }}(\mathrm{ng} \mathrm{h} / \mathrm{mL})$ & $\mathrm{AUC}_{\text {inf }}(\mathrm{ng} \mathrm{h} / \mathrm{mL})$ & $\mathrm{AUC}_{0-24}(\mathrm{ng} \mathrm{h} / \mathrm{mL})$ & $C_{\max }(\mathrm{ng} / \mathrm{mL})$ & $T_{\max }(\mathrm{h})$ \\
\hline \multicolumn{6}{|l|}{ Cycle 1 Day 28} \\
\hline \multicolumn{6}{|l|}{$400 \mathrm{mg}$ qd } \\
\hline$n$ & 3 & 3 & 3 & 3 & 3 \\
\hline Median & 8916 & 10,192 & 8916 & 795 & 4.0 \\
\hline Min/Max & $7756 / 25,942$ & $7947 / 29,223$ & $7769 / 26,182$ & $600 / 1860$ & $4.0 / 4.0$ \\
\hline \multicolumn{6}{|l|}{$800 \mathrm{mg} q d$} \\
\hline$n$ & 3 & 2 & 3 & 3 & 3 \\
\hline Median & 24,870 & 13,540 & 24,955 & 1980 & 2.0 \\
\hline Min/Max & $417 / 26,167$ & $428 / 26,651$ & $417 / 26,167$ & $41.2 / 2190$ & $1.0 / 4.0$ \\
\hline \multicolumn{6}{|l|}{$1000 \mathrm{mg} \mathrm{qd}$} \\
\hline$n$ & 4 & 2 & 2 & 4 & 4 \\
\hline Median & 4804 & 3769 & 3736 & 548 & 4.0 \\
\hline Min/Max & $641 / 11,714$ & $649 / 6889$ & $641 / 6831$ & $153 / 935$ & $1.0 / 6.0$ \\
\hline \multicolumn{6}{|l|}{$1200 \mathrm{mg} \mathrm{qd}$} \\
\hline$n$ & 2 & 0 & 1 & 2 & 2 \\
\hline Median & 37,128 & - & 43,429 & 2170 & 5.9 \\
\hline Min/Max & $31,005 / 43,252$ & - & $43,429 / 43,429$ & $1720 / 2620$ & $3.9 / 8.0$ \\
\hline \multicolumn{6}{|l|}{$1400 \mathrm{mg} \mathrm{qd}$} \\
\hline$n$ & 4 & 3 & 4 & 4 & 4 \\
\hline Median & 18,270 & 30,688 & 18,343 & 1506 & 4.0 \\
\hline Min/Max & $4394 / 36,862$ & $7815 / 45,815$ & $4394 / 36,862$ & $317 / 2510$ & $4.0 / 6.0$ \\
\hline \multicolumn{6}{|l|}{$400 \mathrm{mg}$ bid } \\
\hline$n$ & 4 & 1 & 3 & 4 & 4 \\
\hline Median & 34,083 & 2663 & 29,372 & 3650 & 4.0 \\
\hline Min/Max & $2493 / 80,497$ & $2663 / 2663$ & $2493 / 40,839$ & $518 / 8290$ & $1.0 / 6.0$ \\
\hline
\end{tabular}

$A U C$ area under the curve, bid twice daily, $C_{\max }$ maximum observed serum concentration after drug administration, $P K$ pharmacokinetic, $T_{\max }$ time to reach $\mathrm{C}_{\max }, q d$ once daily

(3/6) treated with BEZ235 at the dose of $1400 \mathrm{mg}$ experienced DLTs including asthenia, fatigue, hyperglycemia, diarrhea, and mucositis (all grade 3). Eventually, $1200 \mathrm{mg}$ was declared as the MTD based on the results from Bayesian Logistic Regression Model (BLRM), in which two DLTs (grade 3 vomiting and grade 2 nausea) were reported in six evaluated patients. Considering the safety profile observed in other clinical studies, $1000 \mathrm{mg}$ was determined as an appropriate RD for further studies [10]. These findings support the results of our study $[11,12]$.

The bid dosing schedule had been introduced with an expectation to improve the absorption, and hence, the daily exposure of BEZ235. In addition, the gastrointestinal toxicities considered to be caused by local irritation of the gastrointestinal tract with qd dosing were expected to be mitigated through the division of the total daily dose. Considering the available strength at that time, only the $400 \mathrm{mg}$ bid dose was investigated in this study, as it was the expected RD for the bid regimen based on the preliminary data from the clinical study in Western oncology patients. In that study, the preliminary results showed that two DLTs were observed at the $600 \mathrm{mg}$ bid dose level, and $400 \mathrm{mg}$ bid was considered as a tolerable dose in this study. Finally, the MTD and RD of BEZ235 in the clinical phase I study in Western oncology patients were declared as $300 \mathrm{mg}$ bid each [12]. In our study, one out of six patients treated with $400 \mathrm{mg}$ bid experienced a DLT of grade 2 liver dysfunction. Although the limited number of bid dose levels evaluated in this study does not allow further conclusions, this result of $400 \mathrm{mg}$ bid is considered to meet the MTD definition as the highest dose level that causes DLT in no more than 1 of 6 patients. Overall, no major differences were observed between the safety profiles of the qd and bid groups. However, gastrointestinal toxicities of grade 3 or 4 , such as nausea, tended to be more frequent in the $400 \mathrm{mg}$ bid group compared with the $800 \mathrm{mg}$ qd group, even though the total daily dose of $800 \mathrm{mg}$ qd and $400 \mathrm{mg}$ bid were the same, and the tolerability of $800 \mathrm{mg}$ qd BEZ235 tended to be better than that of $400 \mathrm{mg}$ bid. No patient in the $800 \mathrm{mg}$ qd group reported dysphagia, but three out of eight patients complained of dysphagia in the $400 \mathrm{mg}$ bid group. This could be because of the study medication being poorly soluble and difficult to swallow at the doses administered 
in this study. The results are similar to the phase I study in Western oncology patients, in which, bid dosing schedule did not result in decreased rates of toxicity compared to qd [12]. Consequently, other clinical studies of BEZ235 with bid dose also showed considerable toxicity [13].

Following oral administration at doses between $400 \mathrm{mg}$ qd and $1400 \mathrm{mg}$ qd, BEZ235 showed high inter- and intrapatient variability with different exposures. Once-daily dosing schedule of multiple formulations including hard gelatin capsule, SDS capsule, and SDS sachet were investigated in a clinical trial in Western oncology patients. However, across all formulations and dose levels tested ( $\geq 100 \mathrm{mg}$ ), BEZ235 displayed low bioavailability, with a moderate absorption rate, a less than proportional increase in exposure, and large inter-patient and intra-patient variability [9]. After bid administration, slow absorption and large inter-patient and intra-patient variability were observed. The variability in PK observed in this study in Japanese oncology patients was consistent with the results from the studies in Western oncology patients. The slow absorption and the low solubility of BEZ235 support a hypothesis of variable bioavailability. Another possibility is the impact of food intake. The FIH study of BEZ235 indicated that fed condition resulted in a higher exposure at 300 to $400 \mathrm{mg} /$ day compared to fasting conditions at the same dose levels [9]. These results indicated that PK of BEZ235 is sensitive to the effect of food. In this study, patients were instructed to take BEZ235 after breakfast but without strict requirements regarding the food contents. Therefore, the difference in food condition between patients and study day might have affected the PK of BEZ235.

No patient achieved CR or PR at either dosing schedule. SD was observed as the best response in 14 out of 27 and two out of eight evaluable patients in the qd and the bid groups, respectively. Analysis of the pS6 status by a well-validated methodology in the $400 \mathrm{mg}$ bid group revealed that $50 \%$ of the patients (3/6) showed a reduction in pS6 after treatment. Two out of three patients showed SD until Cycle 4 or 5 with dose reduction down to $200 \mathrm{mg}$. A tendency toward a more pronounced reduction in $\mathrm{pS} 6$ was observed in patients showing SD over four cycles (data not shown). Similarly, the mTOR inhibitor everolimus was shown to induce changes in pS6 in skin biopsies of patients with advanced solid tumors [14]. These results seemed to indicate a potential use of pS6 as a surrogate marker of pharmacodynamics of BEZ235. However, there is still no data to indicate the correlation of pS6 expression between tumor and skin, and no dose-related changes could be analyzed in this study. Further studies on pS6 in skin as a surrogate marker are necessary.

In conclusion, the MTD based on the standard $3+3$ method was not reached in this study; however, $1200 \mathrm{mg}$ was declared to be the maximum clinically tolerable dose for Japanese oncology patients using a qd dosing schedule. The dose of $1000 \mathrm{mg}$, which was the highest dose that did not cause any DLT, was determined as the RD. In addition, the tolerability of $400 \mathrm{mg}$ BEZ235 with bid dosing was confirmed in Japanese oncology patients. The inter-individual variability of BEZ235 plasma concentrations in Japanese patients was high; however, plasma concentrations were consistent with the range reported in previous studies in Western oncology patients [9]. These results were comparable to the conclusions of the global phase I study of BEZ235.

Although BEZ235 as a single agent and in combination with other antineoplastic agents were investigated in various clinical studies, consideration of the more potent inhibition of mTOR than PI3K and highly variable PK profile of BEZ235 contributed to the discontinuation of further development in oncology indications [9].

Acknowledgements The authors would like to thank the participating patients, their families, all study co-investigators, and research coordinators. The authors thank Takuji Aoki who was involved in the interpretation of study results and drafting of this manuscript. We also thank Rohit Kachhadiya of Novartis Healthcare Pvt. Ltd. for providing medical editorial assistance with this manuscript.

Funding This study was funded by Novartis Pharmaceuticals Corporation.

\section{Compliance with ethical standards}

Conflict of interest Taro Amagasaki, Kazuto Natsume, Hiromi Takeuchi, and Cornelia Quadt are employees of Novartis. Hironobu Minami has received research funding from Novartis KK. The remaining authors declare no competing interests.

Ethical approval All procedures performed in studies involving human participants were in accordance with the ethical standards of the institutional and/or national research committee and with the 1964 Helsinki declaration and its later amendments or comparable ethical standards.

Open Access This article is distributed under the terms of the Creative Commons Attribution 4.0 International License (http://creativeco mmons.org/licenses/by/4.0/), which permits unrestricted use, distribution, and reproduction in any medium, provided you give appropriate credit to the original author(s) and the source, provide a link to the Creative Commons license, and indicate if changes were made.

\section{References}

1. Kang S, Bader AG, Vogt PK (2005) Phosphatidylinositol 3-kinase mutations identified in human cancer are oncogenic. Proc Natl Acad Sci USA 102:802-807

2. Sarbassov DD, Guertin DA, Ali SM, Sabatini DM (2005) Phosphorylation and regulation of Akt/PKB by the rictormTOR complex. Science 307:1098-1101

3. Chow L, Baker S (2006) PTEN function in normal and neoplastic growth. Cancer Lett 241:9817-9824 
4. Ohgaki H (2005) Genetic pathways to glioblastomas. Neuropathology 25:1-7

5. Zeng Z, Samudio IJ, Zhang W et al (2006) Simultaneous inhibition of PDK1/Akt and Fms-like tyrosine kinase 3 signaling by a small-molecule KP372-1 induces mitochondrial dysfunction and apoptosis in acute myelogenous leukemia. Cancer Res 66:3737-3746

6. Maira SM, Stauffer F, Brueggen J et al (2008) Identification and characterization of NVP-BEZ235, a new orally available dual phosphatidylinositol 3-kinase/mammalian target of rapamycin inhibitor with potent in vivo antitumor activity. Mol Cancer Ther 7:1851-1863

7. Burris H, Rodon J, Sharma S et al (2010) First-in-human phase I study of the oral dual PI3K and mTORC1/2 inhibitor BEZ235 in patients with advanced solid tumors. J Clin Oncol 28:3005

8. Rodon J, Infante J, Burris H, Tabernero JM (2010) A dose escalation study with a Special Drug Delivery System (SDS) of BEZ235, a novel dual PI3K/mTOR inhibitor, in patients with metastatic/advanced solid tumors. San Antonio Symposium. San Antonio, USA

9. Rodon J, Pérez-Fidalgo A, Krop IE et al (2018) Phase I/Ib dose escalation and expansion study of BEZ235, a dual PI3K/mTOR inhibitor, in patients with advanced solid tumors including patients with advanced breast cancer. Cancer Chemother Pharmacol 82:285-298

10. Rodon A, Burris HA, Schellens JHM et al (2012) Phase I doseescalation study of the oral dual mTOR/PI3K inhibitor BEZ235, solid dispersion system (SDS) sachet formulation, in patients with advanced solid tumors. Eur J Cancer 48:Suppl 6:112

11. Peyton JD, Rodon Ahnert J, Burris H et al (2011) A dose-escalation study with the novel formulation of the oral pan-class I PI3K inhibitor BEZ235, solid dispersion system (SDS) sachet, in patients with advanced solid tumors. J Clin Oncol 29:3066

12. Bendell JC, Kurkjian C, Infante JR et al (2015) A phase I study of the sachet formulation of the oral dual PI3K/mTOR inhibitor BEZ235 given twice daily (BID) in patients with advanced solid tumors. Invest New Drugs 33:463-471

13. Carlo MI, Molina AM, Lakhman Y (2016) A Phase Ib Study of BEZ235, a Dual Inhibitor of Phosphatidylinositol 3-Kinase (PI3K) andMammalian Target of Rapamycin (mTOR), in Patients With Advanced Renal Cell Carcinoma. Oncologist 21:787-788

14. Owonikoko TK, Ramalingam SS, Miller DL et al (2015) A translational, pharmacodynamic, and pharmacokinetic phase IB clinical study of everolimus in resectable non-small cell lung cancer. Clin Cancer Res 21:1859-1868 